\title{
Dermatite de Contacto Alérgica em Idade Pediátrica: Estudo Retrospectivo
}

\author{
Ana Catarina Cordeiro', Leonor Ramos², Margarida Gonçalo 3 \\ 'Aluna do mestrado integrado em Medicina da Faculdade de Medicina da Universidade de Coimbra, Coimbra, Portugal \\ ${ }^{2}$ Assistente Hospitalar do Serviço de Dermatologia do Centro Hospitalar e Universitário de Coimbra (CHUC), Coimbra, Portugal \\ ${ }^{3}$ Assistente Sénior do Serviço de Dermatologia do Centro Hospitalar e Universitário de Coimbra (CHUC), e Professor Auxiliar \\ convidada da Faculdade de Medicina da Universidade de Coimbra, Coimbra, Portugal
}

RESUMO - Introdução: A dermatite de contacto alérgica em idade pediátrica, habitualmente considerada pouco frequente, tem sido recentemente objecto de numerosas publicações associadas a um aparente aumento da sua prevalência. Tal pode ser devido a exposição e sensibilização mais precoce a alergénios de contacto ou à mais ampla utilização de testes epicutâneos, cruciais ao diagnóstico desta patologia. Métodos: Com o objectivo de avaliar as características e causas da dermatite de contacto alérgica em idade pediátrica, foram analisados os processos clínicos dos doentes com $<18$ anos que efetuaram testes epicutâneos entre Janeiro de 2005 e Dezembro de 2014 na Unidade de Alergologia de Contacto do Serviço de Dermatologia dos HUC. Avaliaram-se os seguintes parâmetros: sexo, idade, história pessoal e familiar de atopia, localização principal da dermatose, testes positivos a alergénios da série básica ou outros e respectiva relevância, hipótese de diagnóstico e diagnóstico final. Resultados: Foram estudados 106 doentes em idade pediátrica (3,6\% do total), 68 do sexo feminino (64,2\%) e 38 do sexo masculino (35,8\%), quatro com idades compreendidas entre os 3 e 5 anos (3,8\%), 28 entre os 6 e 11 anos (26,4\%) e 74 entre os 12 e 17 anos (69,8\%), $52,8 \%$ com antecedentes de atopia e 36,8\% com lesões localizadas na face e/ou pescoço. Dos 106 doentes, 51 tiveram pelo menos um teste positivo (48,1\%), mais frequentemente no sexo feminino por alergia ao níquel (18,9\%). Seguiram-se o cobalto, a p-fenilenodiamina e a mistura de caínas $(4,7 \%)$ entre os alergénios de série básica e, fora dela, o paládio positivo em cinco crianças. De notar que não observámos testes positivos ao níquel nos últimos dois anos. Doze doentes reagiram apenas a alergénios não incluídos na série básica, sobretudo medicamentos. Discussão: A alergia de contacto e dermatite de contacto alérgica antes dos 18 anos revelaram-se relativamente comuns. O diagnóstico etiológico precoce é importante para evitar a cronicidade das lesões e prevenir possíveis repercussões futuras a nível pessoal e profissional. O conhecimento dos principais alergénios nesta faixa etária poderá orientar medidas preventivas de futuras sensibilizações.

PALAVRAS-CHAVE - Alergénios; Criança; Dermatite Alérgica de Contacto; Níquel; Testes Epicutâneos.

\section{Allergic Contact Dermatitis in the Pediatric Age: A Retrospective Study}

ABSTRACT - Introduction: Allergic contact dermatitis in children, although usually considered infrequent, has been the object of numerous recent publications and an apparent increase in prevalence. This may be due to early exposure and sensitization to contact allergens, increasing the likelihood of skin reactions in a new exposure, or the more widespread use of patch tests, which are crucial to the diagnosis of this pathology. Methods: In order to know the characteristics and causes of allergic contact dermatitis in the pediatric age, we evaluated the medical records of patients with < 18 years who were patch tested between January 2005 and December 2014 at the Contact Allergology Unit of the Department of Dermatology University Hospital of Coimbra. The following parameters were analyzed: sex, age, personal and familiar history of atopy, main location of the dermatitis, reactive allergens within the baseline or complementary test series, suspected and final diagnosis. Results: A total of 106 pediatric patients were tested (3.6\% of all patients), 68 females (64.2\%) and 38 males (35.8\%), four aged between 3-5 years (3.8\%), 28 between 6- 11 years (26.4\%)

Correspondência: Margarida Gonçalo

Serviço de Dermatologia dos CHUC - Praceta Mota Pinto, 3000-075 Coimbra

Tel.: + 351.239400420

E-mail:mgoncalo@fmed.uc.pt
Recebido/Received

12 Abril/April 2017

Aceite/Accepted

21 Maio/May 2017 


\section{Artigo Original}

and 74 between $12-17$ years (69.8\%), 52,8\% with a personal history of atopy, 36.8\% with facial and/or neck lesions. At least one positive test was observed in 51 patients (48.1\%), mostly in girls especially because of nickel (18.9\%). Nevertheless there were no positive tests to nickel in the last two years of the study. After nickel, cobalt, p-phenylenediamine, caine mix and palladium were reactive in five patients. Twelve patients reacted to allergens that are not included in the baseline series, mostly to drugs. Discussion: Contact allergy and allergic contact dermatitis were a relatively common diagnosis before the age of 18. An early etiologic diagnosis is important to prevent chronicity of lesions and a possible future negative personal and professional impact. Knowing the main allergens in this age group is important to establish avoidance measures and prevent further sensitization.

KEYWORDS - Allergens; Children; Dermatitis, Allergic Contact; Nickel;Patch Tests.

\section{INTRODUÇÃO}

A dermatite de contacto alérgica (DCA) era considerada rara na infância, existindo a noção de que as crianças seriam menos expostas a alergénios de contacto e que o seu sistema imune, por mais imaturo, seria menos susceptível à sensibilização. Contudo, nos últimos anos esse facto tem vindo a ser contestado com um número crescente de casos de DCA descritos em crianças e adolescentes. ${ }^{1}$ Este facto pode também estar relacionado com a inclusão de doentes com um leque de idades cada vez maior, atualmente até aos 18 anos. A segurança dos testes epicutâneos em crianças mesmo nos primeiros anos de vida está comprovada pelo que o seu uso se tem generalizado no diagnóstico etiológico de DCA também nesta faixa etária. ${ }^{2}$

Nos adultos, a frequência de testes epicutâneos positivos na população normal varia entre $10-27 \%$ da população, ${ }^{3}$ mas a exata prevalência e incidência nas crianças é ainda desconhecida e difícil de quantificar. ${ }^{4-6}$ Sendo uma causa frequente de eczema ou de agravamento de dermatoses pré-existentes, a DCA pode ter grande impacto a nível pessoal e socioeconómico bem como implicações na vida adulta e no futuro profissional destas crianças.

\section{OBJETIVO}

O objetivo principal deste trabalho foi avaliar as características e causas da DCA em crianças e adolescentes ao longo de 10 anos no sentido de contribuir para uma melhor percepção da importância e impacto da DCA nesta faixa etária, bem como detetar algumas modificações ao longo dos últimos anos.

\section{MATERIAL E MÉTODOS}

De um total de 2909 doentes que efetuaram testes epicutâneos entre Janeiro de 2005 e Dezembro de 2014 na Unidade de Alergologia de Contacto do Serviço de Dermatologia dos CHUC, foram analisados retrospectivamente os processos clínicos dos doentes testados em idade pediátrica (< 18 anos).

Todas as crianças foram submetidas a testes epicutâneos com os alergénios da série básica adoptada pelo Grupo Português de Estudo de Dermatites de Contacto (GPEDC) com adições locais e, sempre que necessário com séries complementares ou alergénios específicos (Chemotechnique Diagnostics ${ }^{\circledR}$, Vellinge, Suécia; Trolab Allergens ${ }^{\circledR}$, Smartpractice $\mathrm{GmBH}$, Alemanha; Bial Alergénios) ou ainda com "produtos próprios". Pontualmente houve necessidade de reduzir o número de alergénios testados por limitação de espaço no dorso das crianças de menor idade. Os alergénios foram colocados em câmaras de alumínio de $8 \mathrm{~mm}$ (Finn Chambers ${ }^{\circledR}$ in Scanpor tape, Smartpractice Europe $\mathrm{GmBH}$, Alemanha), aplicados na parte superior do dorso e mantidos em oclusão durante 48 horas. A primeira leitura foi realizada no dia 2 (D2) ou D3 e a segunda leitura entre D4 e D7, utilizando os critérios do International Contact Dermatitis Research Group e da European Society of Contact Dermatitis. ${ }^{2}$

Foram avaliados os seguintes parâmetros: sexo, idade, história pessoal ou familiar de atopia - eczema atópico, asma e/ou rinite alérgicas -, localização principal da dermatite, hipótese de diagnóstico, resultados dos testes, dentro e/ou fora da série básica de alergénios, relevância das reações positivas e diagnóstico final. Foram consideradas positivas as reacções com eritema e infiltração $(1+)$ ou mais intensas. Alguns dados da população pediátrica foram comparados com a população total de doentes testados no mesmo serviço e no mesmo período de tempo.

$\mathrm{Na}$ avaliação dos resultados utilizou-se o SPSS-Statistics versão 20.0. Foram determinadas estatísticas descritivas, medidas de tendência central e de dispersão. ${ }^{7}$ A comparação de proporções foi feita através do teste do X2 (qui-quadrado). Utilizou-se também um teste não paramétrico, o teste de Kruskal-Wallis (para três ou mais amostras independentes). $O$ valor $p$ igual ou inferior a 0,05 foi considerado significativo.

\section{RESULTADOS \\ 1. Caracterização dos doentes estudados em idade pediátrica}

Entre Janeiro 2005 e Dezembro 2014 (10 anos) foram estudados 2909 doentes dos quais $106(3,6 \%)$ com idades compreendidas entre os 3 e os 17 anos (média 13,01 $\pm 3,61$ anos), 68 do sexo feminino $(64,2 \%)$ e 38 do sexo masculino $(35,8 \%)$. Foram considerados três grupos etários, com quatro doentes entre os 3 e os 5 anos (3,8\%), 28 entre os 6 e os 11 anos $(26,4 \%)$ e 74 entre os 12 e os 17 anos $(69,8 \%)$ (Tabela 1). Neste período, o número de casos pediátricos testados por ano rondou os $3 \%$, destacando-se o ano de 2010 em que o total de casos representou $6,8 \%$ do total de consultas.

Cinquenta e seis doentes $(52,8 \%)$ tinham manifestações de atopia, $36 \mathrm{com}$ eczema atópico $(34,0 \%), 22 \mathrm{com}$ asma $(20,8 \%)$ e 23 com rinite alérgica $(21,7 \%)$, sobretudo no sexo 


\section{Artigo Original}

Tabela 1 - Características da população testada em relação à idade, sexo e atopia.

\begin{tabular}{c|c|c|c}
\multirow{2}{*}{ Idade } & & n com atopia & com atopia \\
\cline { 2 - 4 } & $3-5 \operatorname{anos}(\mathrm{n}=4)$ & 1 & $25 \%$ \\
\cline { 2 - 4 } & $6-11 \operatorname{anos}(\mathrm{n}=28)$ & 16 & $57,1 \%$ \\
\hline \multirow{2}{*}{ Sexo } & $12-17 \operatorname{anos}(\mathrm{n}=74)$ & 39 & $52,7 \%$ \\
\cline { 2 - 4 } & Feminino( $\mathrm{n}=68)$ & 39 & $57,4 \%$ \\
\hline \multirow{2}{*}{ Total } & Masculino(n=38) & 17 & $44,7 \%$ \\
\hline
\end{tabular}

Tabela 2 - Localização das lesões consoante os grupos etários.

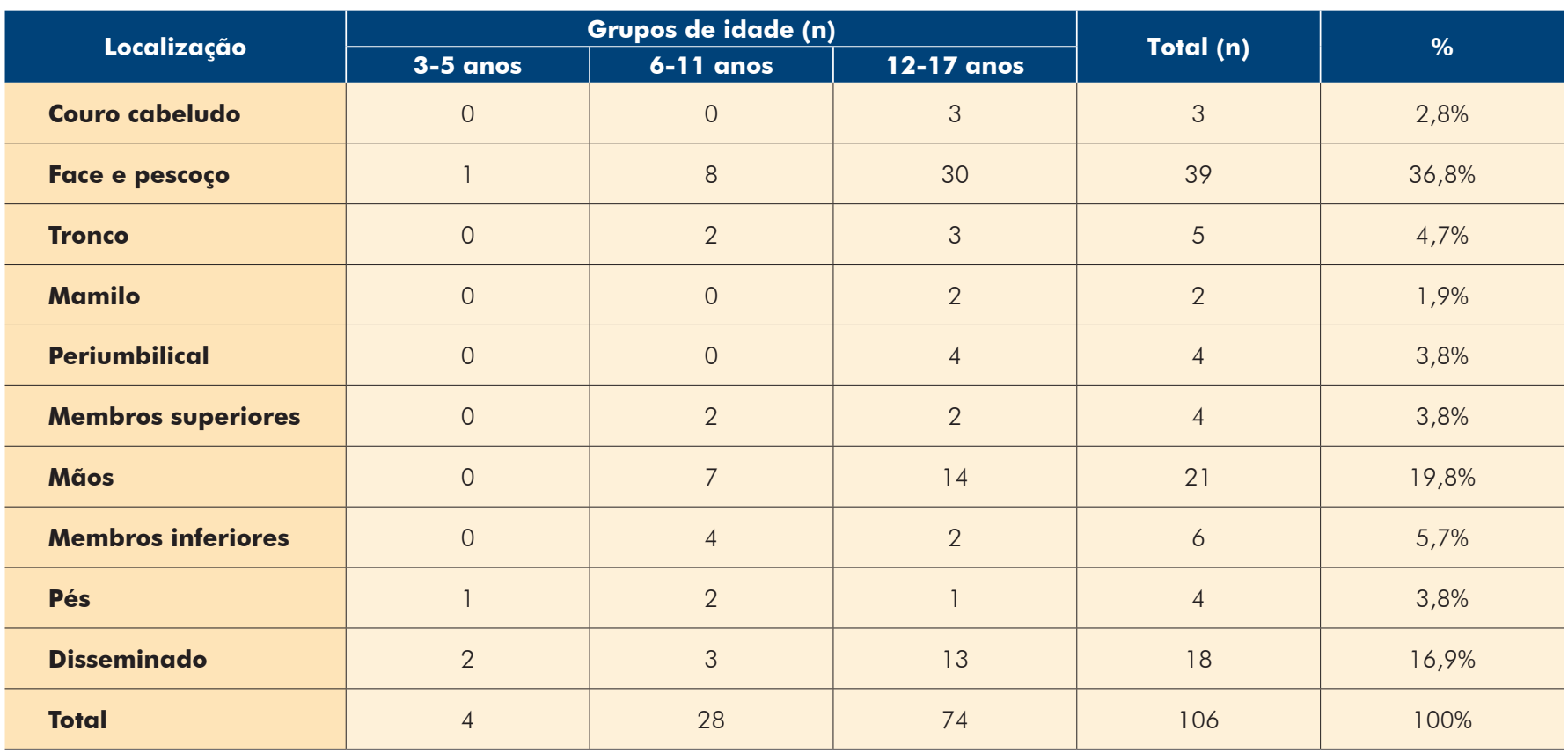

feminino $(57,4 \%)$ e entre os 6 e os 11 anos $(57,1 \%)$ (Tabela 1$)$.

Os testes epicutâneos foram realizados maioritariamente para esclarecimento de suspeita de eczema de contacto (92), em 28 casos em associação com eczema atópico, em três com eczema desidrótico e em 16 casos para estudo de exantema considerado medicamentoso.

As localizações mais frequentes das lesões foram face e/ou pescoço $(36,8 \%)$, seguidos das mãos $(19,8 \%)$, não se verificando diferença significativa da localização nos diferentes grupos etários ( $p>0,05)$ (Tabela 2).

\section{Reatividade dos testes epicutâneos em idade} pediátrica

Dos 106 doentes, 51 tiveram pelo menos um teste positivo $(48,1 \%)$, numa percentagem inferior à dos doentes com idade igual ou superior a 18 anos testados no mesmo período na consulta $(69,1 \%)$. Dos 51 doentes, 23 reagiram apenas a um alergénio (45\%), 10 a dois $(19,6 \%), 18$ a três ou mais alergénios $(35,4 \%)$, e 12 doentes $(23,5 \%)$ reagiram apenas a alergénios não pertencentes à série básica.

A reactividade foi significativamente maior no sexo feminino $(53,4 \%$ versus $31,6 \%$; $p<0,05)$ e nos pacientes com atopia $(55,4 \%)$ mas, semelhante nos três grupos etários estudados $(p>0,05)$. A idade média dos pacientes com testes positivos $(13,29 \pm 3,45)$ e testes negativos $(12,75 \pm 3,70)$ não foi significativamente diferente (Tabela 3 ).

\section{Principais alergénios positivos}

Os principais alergénios da série básica responsáveis por provas positivas (Tabela 4) foram o sulfato de níquel (20), seguido do cloreto de cobalto, da parafenilenodiamina e da mistura de caínas (cinco cada) e do timerosal (quatro). 


\section{Artigo Original}

Tabela 3 - Resultado dos testes epicutâneos consoante os grupos etários, sexo e associação com atopia.

\begin{tabular}{|c|c|c}
\hline Grupos etários* & Testes positivos & Testes negativos \\
\hline $\mathbf{3 - 5}$ anos $(\mathbf{n}=\mathbf{4})$ & $50 \%(\mathrm{n}=2)$ & $50 \%(\mathrm{n}=2)$ \\
\hline $\mathbf{6 - 1 1}$ anos $(\mathbf{n}=\mathbf{2 8})$ & $42,9 \%(\mathrm{n}=12)$ & $57,1 \%(\mathrm{n}=16)$ \\
\hline $\mathbf{1 2 - 1 7}$ anos $(\mathbf{n}=\mathbf{7 4})$ & $50 \%(\mathrm{n}=37)$ & $50 \%(\mathrm{n}=37)$ \\
\hline Feminino $(\mathbf{n}=\mathbf{6 8})$ & $\mathbf{S e x} * *$ & $42,6 \%(\mathrm{n}=29)$ \\
\hline Masculino $(\mathbf{n}=\mathbf{3 8})$ & $53,4 \%(\mathrm{n}=39)$ & $68,4 \%(\mathrm{n}=26)$ \\
\hline Com Atopia $(\mathbf{n}=\mathbf{5 6})$ & $31,6 \%(\mathrm{n}=12)$ & $\mathbf{A t o p i a}$ \\
\hline Sem Atopia $(\mathbf{n}=\mathbf{5 0})$ & $55,4 \%(n=31)$ & $44,6 \%(\mathrm{n}=25)$ \\
\hline
\end{tabular}

* diferença não significativa $(p>0,05)$

** diferença significativa $(p<0,05)$

Tabela 4 - Alergénios reativos dentro da série básica. Número e percentagem de doentes com teste positivo consoante o sexo e comparativamente com toda a população estudada em igual período

\section{Alergénio Reactivo na Série Básica}

\section{Pacientes em Idade Pediátrica}

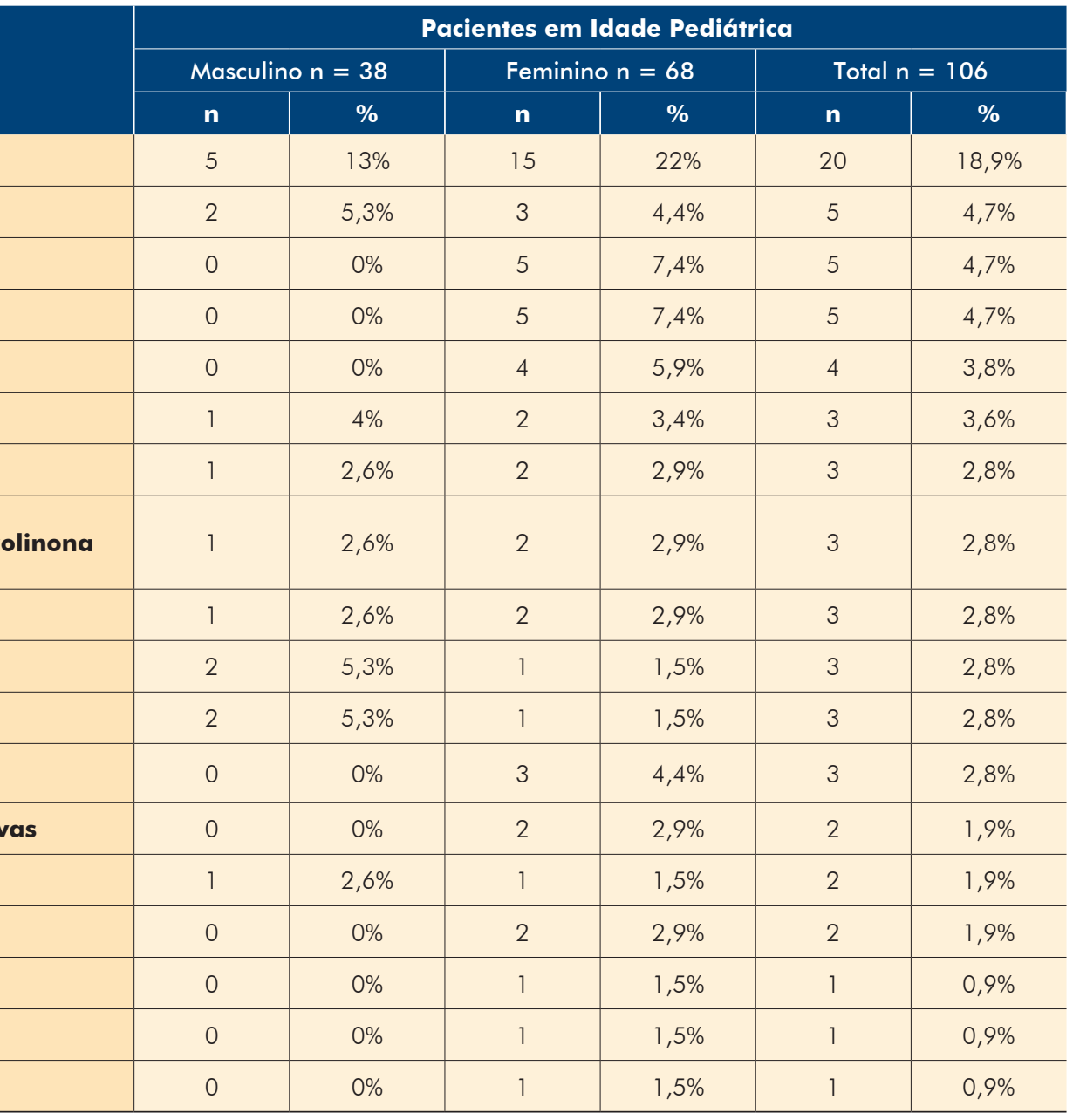

Sulfato de níquel $5 \%$ vas

Cloreto de cobalto $1 \%$ vas

p-fenilenodiamina $1 \%$ vas

Mistura de caínas III - $10 \%$ vas

Timerosal 0,1\%vas

Cloreto de mercúrio amoniacal

Mistura de Perfumes $8 \%$ vas

Metilsotiazolinona 500ppm ág e/ou

Metilisotiazolinona/ clorometilsotiazolinona 100 ppm ág

\section{Formaldeído $1 \%$ ág}

Diazolidinilureia $2 \%$ ag

Imidazolidinilureia $2 \%$ vas

Laranja disperso 3 - $1 \%$ vas

Isopropil - p-fenilenodiamina $0,1 \%$ vas

Dicromato de potássio $0,5 \%$ vas

Amerchol L101 50\% vas

Álcoois de lanolina $30 \%$ vas

Quartenium 15 - 1\% vas

Bálsamo de Perú - 15\% vas 


\section{Artigo Original}

Entre os outros alergénios destacam-se o cloreto de paládio (cinco), a p-tolvenodiamina, o Zovirax creme ${ }^{\circledR}$ e o verniz das unhas (três cada) (Tabela 5).

A reatividade ao níquel (18,9\%) foi significativamente superior no sexo feminino $(15 / 68-22,1 \%)$ relativamente ao sexo masculino (5/38 - 13,2\%) e na faixa etária dos 12 aos 17 anos (16/39 - 41\%). Os restantes casos reativos ao níquel ocorreram entre os 6 aos 11 anos (4/16 - 25\%) sem nenhum teste positivo no grupo mais jovem. A frequência na reatividade ao níquel na idade pediátrica manteve-se semelhante ao longo de 8 anos mas, nos últimos dois anos, não se registou qualquer teste positivo a este alergénio. A reatividade ao níquel nesta população pediátrica foi inferior à da população de todas as faixas etárias testada em igual período no mesmo serviço $(26,9 \%)$.

O cloreto de cobalto foi positivo em cinco crianças (2M) $3 \mathrm{~F})$, em simultâneo com o níquel em 4 delas. A p-fenilenodiamina (PPD) com cinco testes positivos $(4,7 \%)$ foi reactiva

Tabela 5 - Testes epicutâneos positivos fora da série básica.

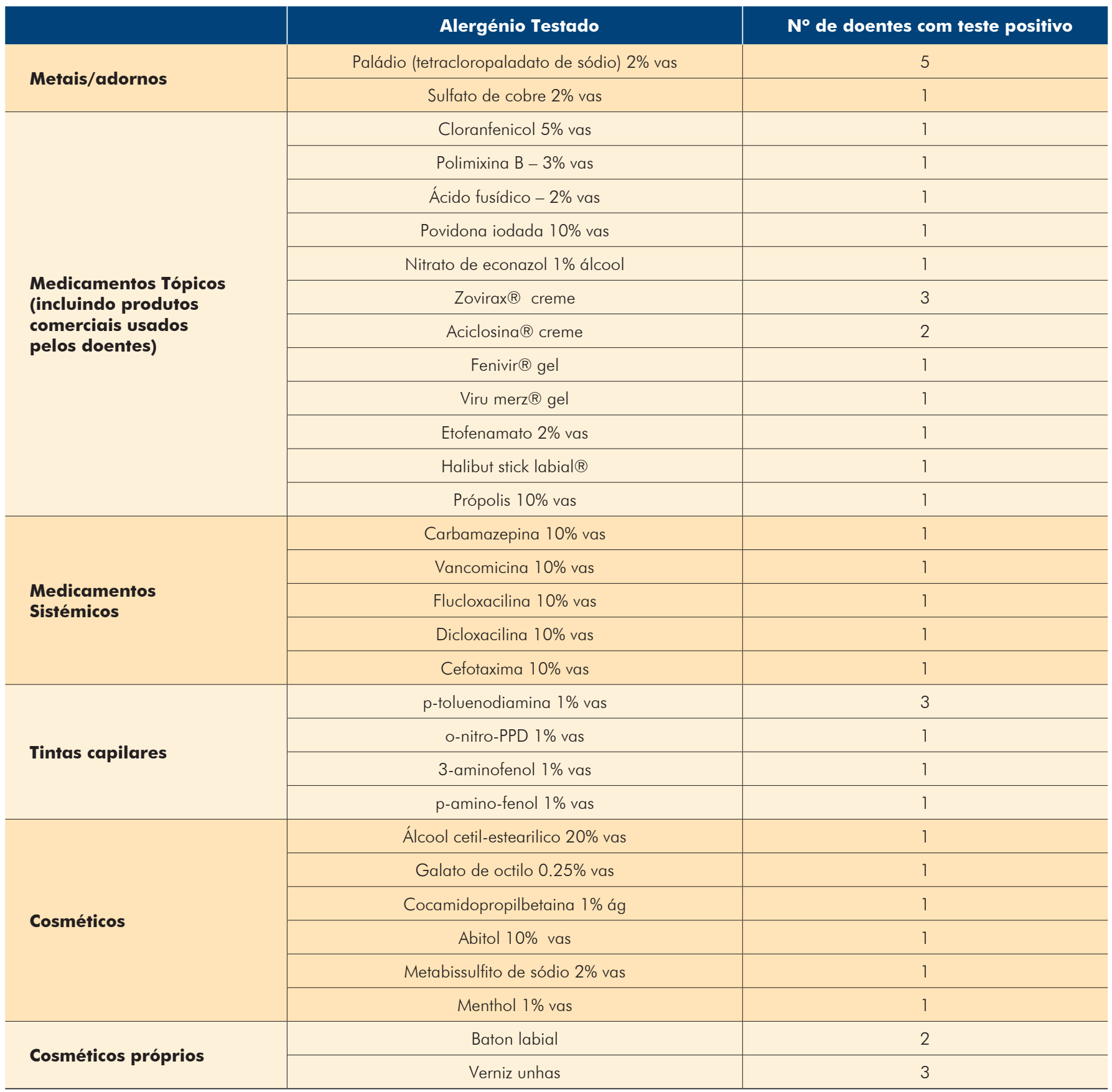




\section{Artigo Original}

Tabela 6 - Testes positivos na série básica e relevância atual ou passada.

\begin{tabular}{|c|c|c|c|}
\hline Alergénio testado & Testes positivos $(n)$ & Reações relevantes $(\mathbf{n})^{*}$ & Causas das reações \\
\hline Níquel & 20 & 18 & \multirow{2}{*}{$\begin{array}{l}\text { Adornos, peças vestuário, aparelho de } \\
\text { ortodôncia (2) }\end{array}$} \\
\hline Cobalto & 5 & 4 & \\
\hline p-fenilenodiamina (PPD) & 5 & 4 & $\begin{array}{c}\text { Tintas capilares (3) e/ou Tatuagem } \\
\text { temporária (2) }\end{array}$ \\
\hline Mistura de caínas III & 5 & 0 & 3 RX com PPD \\
\hline Laranja disperso 3 & 3 & 3 & $\begin{array}{c}\text { Tintas capilares (3) possível RX com } \\
\text { PPD }\end{array}$ \\
\hline $\begin{array}{l}\text { Isopropil-p- } \\
\text { fenilenodiamina }\end{array}$ & 2 & 0 & $\begin{array}{c}\text { RX como PPD por alergia a tintas } \\
\text { capilares }\end{array}$ \\
\hline Timerosal & 4 & 0 & Relação possível com vacinação \\
\hline Formaldeído & 3 & \multirow{4}{*}{3} & \multirow{4}{*}{$\begin{array}{l}\text { Conservantes à base de formaldeído } \\
\text { presentes em cosméticos }\end{array}$} \\
\hline Diazolidinilureia & 3 & & \\
\hline Imidazolidinilureia & 3 & & \\
\hline Quartenium 15 & 1 & & \\
\hline Mistura de perfumes & 3 & 1 & Desodorizante \\
\hline MI ou $\mathrm{MCl} / \mathrm{MI}$ & 3 & 3 & $\begin{array}{c}\text { Cosméticos e produtos de higiene } \\
\text { pessoal }\end{array}$ \\
\hline Crómio & 2 & $1 ?$ & Dispositivo de proteção no futebol \\
\hline Amerchol L101 & 2 & \multirow{2}{*}{2} & \multirow{2}{*}{$\begin{array}{l}\text { Medicamentos tópicos e/ou } \\
\text { cosméticos }\end{array}$} \\
\hline Álcoois de lanolina & 1 & & \\
\hline Bálsamo de Perú & 1 & 1 & Medicamentos tópicos \\
\hline Mercúrio amoniacal & 1 & 0 & RX timerosal \\
\hline
\end{tabular}

*foram consideradas tanto as relevâncias atuais como as passadas; RX - reações cruzadas; MI - metilisotiazolinona;

$\mathrm{MCl} / \mathrm{Ml}$ - mistura clorometilisotiazolinona/metilsiotiazolinona (3:1)

apenas no sexo feminino. A mistura de caínas III, contendo benzocaína, tetracaína e cinchocaína, foi positiva em cinco casos, todos do sexo feminino, em três associada à PPD. Obtivemos quatro testes positivos ao timerosal e três ao mercúrio amoniacal, mas estes alergénios não foram testados nos últimos 2 anos. Destacam-se entre os conservantes da série básica, três casos positivos à metilisotiazolinona (MI) testada apenas nos últimos 2 anos do estudo, à mistura de metilcloroisotiazolinona com $\mathrm{MI}(\mathrm{MCl} / \mathrm{MI})$ (três), ao formaldeído (três) e/ou seus libertadores: imidazolidinilureia (três), diazolidinilureia (três) e quaternium 15 (um).

Não encontrámos testes positivos à neomicina, azul disperso 106, mistura de parabenos, metildibromoglutaronitrilo (dibromodicianobutano), resina epóxida, resina formol-butil-fenol-para-terciária, mercaptobenzotiazol, misturas de mercapto, tiurans ou carbamatos, colofónia, mistura de lactonas, pivalato de tixocortol, budesonido, 17-butirato de hidrocortisona e hidroxisohexyl 3-ciclohexeno carboxaldeído (Lyral ${ }^{\circledR}$ ).
Dos alergénios não incluídos na série básica, destaca-se o cloreto de paládio em cinco casos (4F/1M), reactivo sempre em simultâneo com o níquel, e a p-tolvenodiamina e outros alergénios de tintas capilares (três), em simultâneo com aPPD. Em 12 doentes ocorreram testes positivos exclusivamente fora da série básica de alergénios e estes testes foram determinantes para o diagnóstico etiológico, nomeadamente no caso de reações a medicamentos tópicos ou sistémicos (Tabela 5).

\section{Relevância das reações positivas}

Da análise da relevância, destacamos que a grande maioria dos testes positivos tinham relevância atual ou passada. Os casos de reações positivas múltiplas eram explicáveis por reações cruzadas ou sensibilização concomitante, como nos cinco casos por metais (níquel, paládio e cobalto), cinco casos por alérgenios do grupo para-amino (PPD, mistura de caínas, isopropil-PPD, Laranja disperso três e p-tolvenodiamina) e três 


\section{Artigo Original}

entre o formaldeído e os seus libertadores (imidazolidinilureia, diazolidinilureia e quaternium 15).

As reações ao níquel, cobalto e paládio, relevantes em $90 \%$ dos casos, na sua maioria com relevância passada (dez), estavam relacionadas com adornos ou peças metálicas do vestuário (seis) ou material de ortodôncia (dois) (Fig.s 1 e 2). Quatro das cinco reações positivas à PPD estavam relacionadas com tatuagens temporárias (Fig.s 3 e 4) e/ou tintas capilares, mas sem relação com a atividade profissional. Nas diferentes reações positivas a perfumes e conservantes estes constituintes foram frequentemente identificados em medicamentos tópicos, cosméticos ou produtos de higiene pessoal.

Dos 59 pacientes com suspeita de eczema de contacto identificámos o agente responsável em 30 (50,8\%), destacando os casos por medicamentos tópicos (antifúngicos e antivíricos), cosméticos e metais (cintos, brincos e peças de vestuário). Em sete dos 36 doentes com eczema atópico $(19,4 \%)$ foi também identificado um alergénio relevante. Em 28 casos $(26,4 \%)$ não foram identificados alergénios de contacto no teste epicutâneo nem obtivemos reações ao material do doente testado

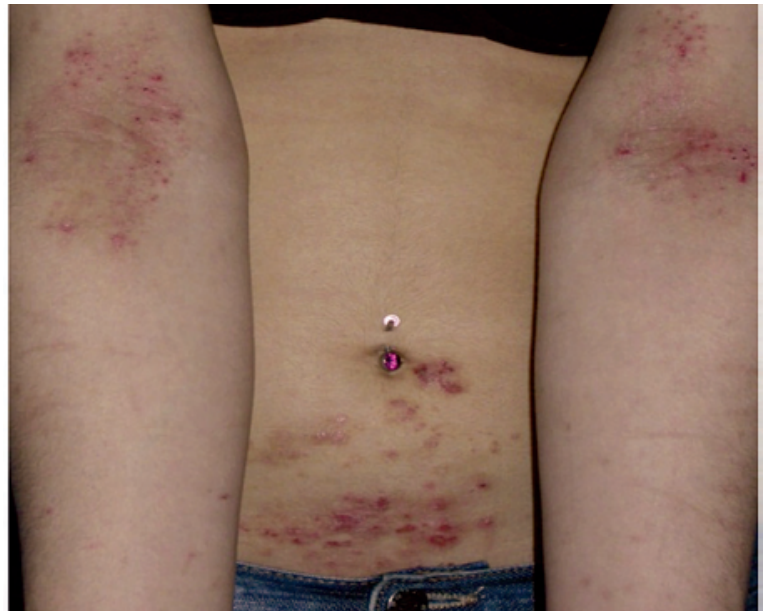

Figura 1 - DCA ao níquel em criança com eczema atópico

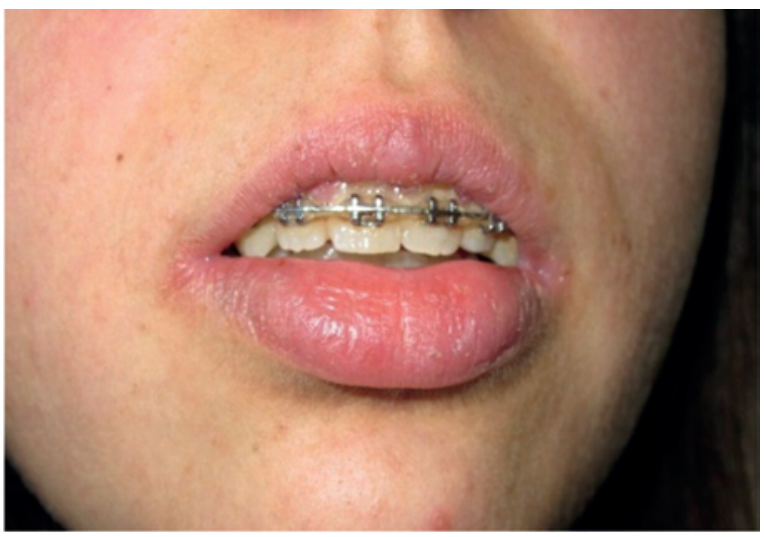

Figura 2 - Queilite após utilização de aparelho de ortodôncia em doente com testes positivos ao níquel.
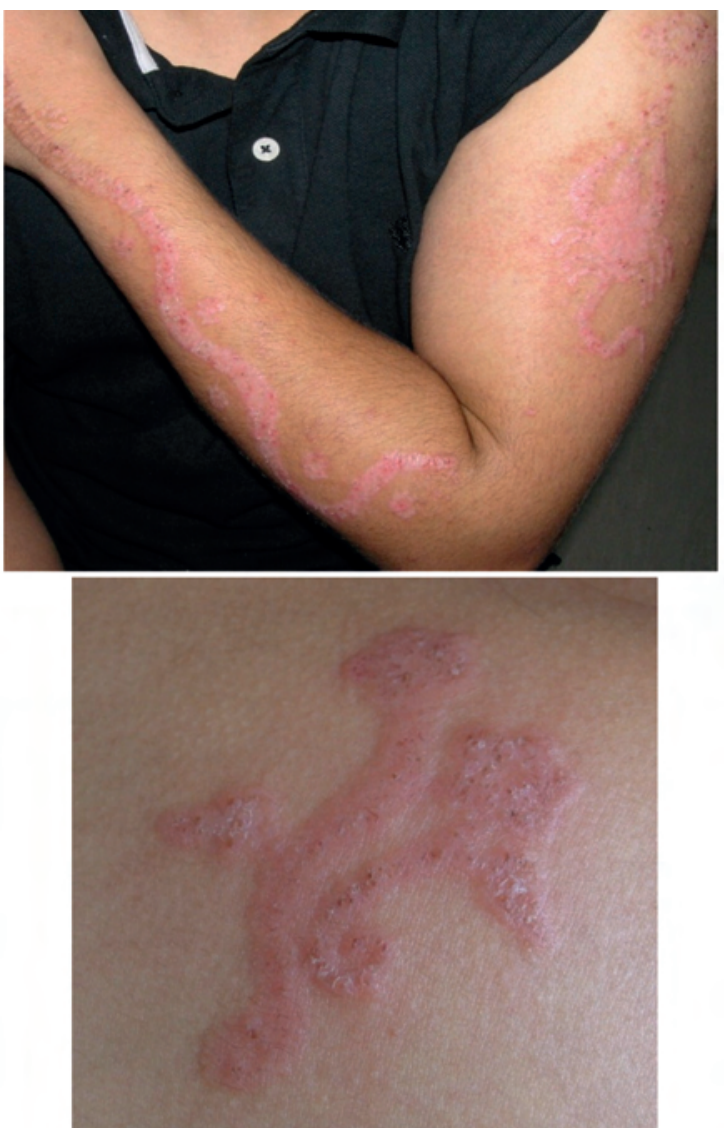

Figura 3 e 4 - DCA a tatuagem provisória em fase subaguda.

tal qual, nomeadamente em quatro jovens com eczema periocular que relacionavam com o uso de óculos de proteção para a natação (Fig. 5), três com eczema da face anterior das

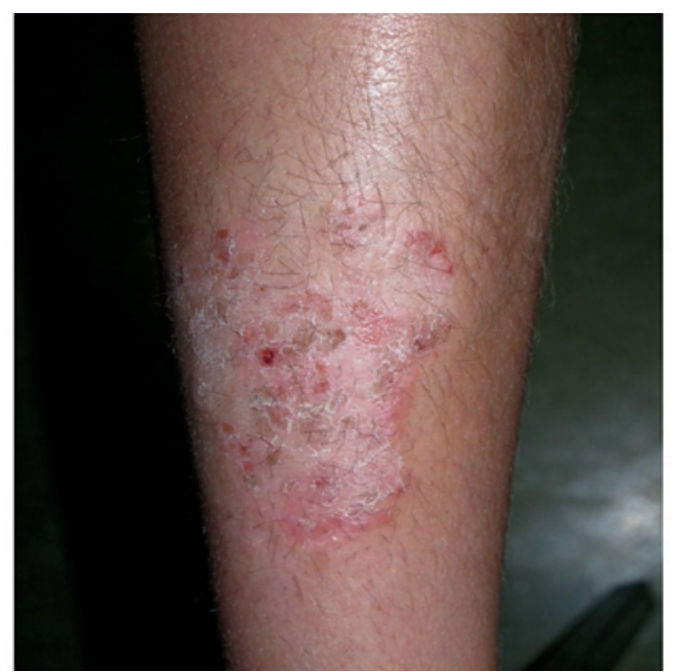

Figura 5 - Eczema de contacto da face anterior das pernas desenhando a área de contacto com as caneleiras de protecção utilizadas no futebol, mas em que não foi identificado qualquer alergénio. 


\section{Artigo Original}

pernas desenhando o contacto com as caneleiras de proteção do futebol (Fig. 6) e dois com dermatite das mãos que atribuíam às luvas de guarda-redes.

Em 13 jovens com suspeita de exantema medicamentoso foi possível confirmar a imputabilidade clínica em quatro casos $(30,8 \%)$, nomeadamente à carbamazepina, cefotaxima, vancomicina e flucloxacilina, este último também com teste positivo à dicloxacilina.

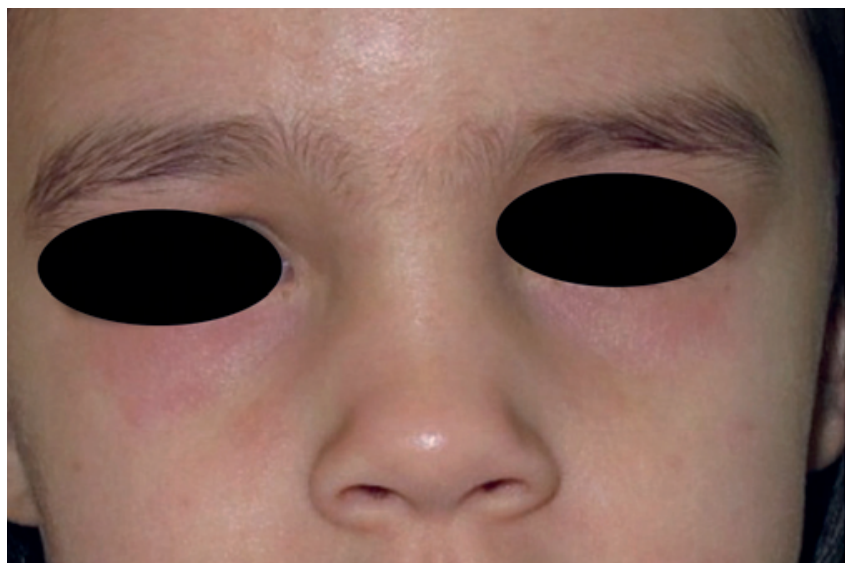

Figura $\mathbf{6}$ - Eczema periocular relacionado com exposição a óculos de natação em borracha ou silicone.

\section{DISCUSSÃO}

Alguns estudos referem a DCA como uma patologia relativamente comum nas crianças e com aumento de prevalência com a idade. ${ }^{1,8-10}$ No presente estudo, os 106 doentes testados em idade pediátrica com suspeita de DCA ou outra reação de hipersensibilidade retardada representaram uma pequena fração $(3,6 \%)$ do total de doentes testados no mesmo período e a maioria tinha entre os 12 e os 17 anos, Estes valores apoiam a noção de que a DCA aumenta com a idade, contudo, a percentagem de testes positivos nos diferentes grupos etários foi semelhante. Apesar de ter sido verificada a segurança destes testes em crianças, logo após os 6 meses de idade, ${ }^{11}$ o reduzido número de crianças testadas com menor idade pode ser também devido a maior relutância em submeter estas crianças à realização de testes, consequentemente limitando a sua realização às crianças com reações mais graves e/ou recidivantes.

No presente estudo, as localizações mais frequentes da DCA foram a face, o pescoço e as mãos $(56,6 \%)$ tal como noutros estudos. ${ }^{12}$ Porém, a localização aos pés, considerada frequente na criança, ${ }^{13,14}$ ocorreu apenas em quatro casos e, não encontrámos qualquer reação a constituintes do calçado como o crómio, a resina de butil-fenol-para-terciário ou os alergénios das borrachas, frequentemente referidos como causa de DCA dos pés em crianças. $4,12,13$

É ainda controverso se os doentes com atopia têm maior ou menor propensão para DCA. ${ }^{15,17}$ Neste estudo, a positividade dos testes epicutâneos é maior nos doentes com atopia pessoal $(55,4 \%)$ o que pode sugerir que estes doentes podem

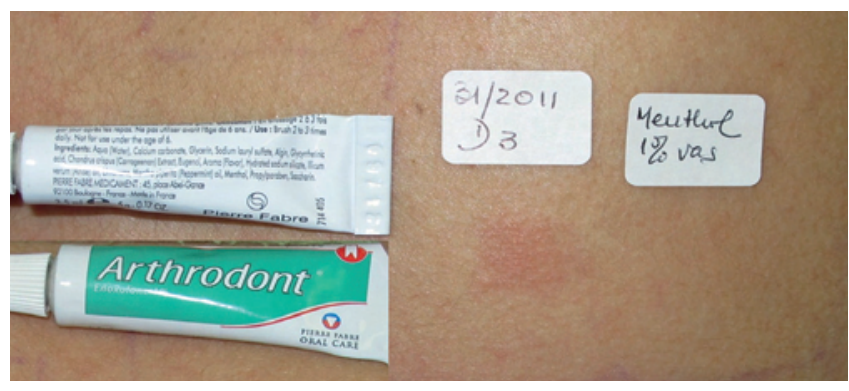

Figura 7 - Teste epicutâneo positivo a mentol, componente da pasta de dentes Arthrodont utilizada pelo doente.

ter um risco aumentado de desenvolver DCA ou maior exposição a medicamentos tópicos e emolientes, sobretudo no caso do eczema atópico.

Cerca de dois terços das crianças eram do sexo feminino e nestas foi maior a percentagem de reações positivas (F:53,4\% vs $M: 31,6 \%$, tal como noutros estudos publicados, ${ }^{15,16}$ sobretudo à custa do níquel a que as crianças do sexo feminino estão mais expostas.

O níquel, alergénio muito frequente $(18,9 \%)$ como em muitos outros estudos, $1,9,11-13,15,16,19-21$ teve uma percentagem de reações positivas semelhante ao longo dos 8 anos iniciais do estudo mas sem qualquer reação em 2013 ou 2014. Ainda que se possa tratar de um achado ocasional, ele vai ao encontro de dados obtidos em estudo recente de alergia a metais na mesma consulta: a percentagem de jovens entre os indivíduos sensibilizados ao níquel diminuiu nos últimos anos. ${ }^{22}$ Assim, e ainda que tardiamente, é muito provável que esteja a ser implementada em Portugal a Diretiva da Comunidade Europeia (1994/27/CE;2004/96/CE) relativamente aos limites no conteúdo e libertação de níquel de objetos em contacto prolongado com a pele (bijutaria, relógios, fivelas de cintos, telemóveis, etc.) com redução da sensibilização das gerações mais jovens. Com a aplicação ativa destas restrições nos países da Europa do Norte, verificou-se uma redução significativa da percentagem de indivíduos sensibilizados ao níquel na população normal ${ }^{23}$ e uma redução dos casos de DCA ao níquel entre os jovens, diferenças de prevalência bem patentes em estudo recente na população geral $>18$ anos entre os países do Norte (Suécia) e os países do Sul (Itália e Portugal) onde estas medidas têm sido menos reforçadas: $18,5 \%$ e $16,4 \%$ de alergia de contacto ao níquel respectivamente em Portugal e Itália e $8,3 \%$ na Suécia. ${ }^{3}$ Contudo, não é só no nosso país que estas medidas não são ativa e completamente implementadas: recentemente na Alemanha verificou-se que uma percentagem não desprezível de brinquedos ou objetos de construção civil libertavam quantidades apreciáveis de níquel. ${ }^{24}$

Os sais de cobalto e paládio, reativos simultaneamente com o níquel, tal como habitualmente descrito, podem coexistir em peças de bijutaria, relógios e adornos do vestuário e ligas metálicas usadas em material dentário e, sensibilizar o indivíduo de forma concomitante. Mas estes metais também desencadeiam a mesma resposta inata (ativação de Toll-like receptor-4 (TLR4) ${ }^{28}$ e podem ser reconhecidos por receptores 


\section{Artigo Original}

de células T (TCR) comuns (reações cruzadas). Foram relativamente raras as reações isoladas ao cobalto e crómio. O crómio causa habitualmente DCA ao calçado em pele, mas não observámos casos de DCA ao calçado ou outros objectos em pele.

A p-fenilenodiamina é causa frequente de testes positivos $(9,8 \%)$, relacionados com tintas capilares e tatuagens provisórias em que ao extrato de Henna é adicionado PPD em concentrações elevadas. ${ }^{4,13,26,27}$ Estas tatuagens temporárias causam sensibilização ativa e DCA exuberantes, ${ }^{13}$ e frequentemente reações à aplicação de tintas capilares, com lesões edematosas e erisipeloides da face e pescoço que se confundem com o angioedema e podem comprometer as vias aéreas superiores. ${ }^{29}$ Ainda, esta sensibilização acarreta um risco acrescido de reações cruzadas a químicos do grupo para-, como outros constituintes de tintas capilares ( $p$-tolvenodiamina), corantes têxteis, anestésicos locais (benzocaína), observados no presente estudo, e ainda filtros solares (ácido para-amino-benzoico/PABA) e sulfamidas. ${ }^{26}$

A positividade a fragrâncias (mistura de perfumes I e II e bálsamo do Perú) é também comum na idade pediátrica $(2,8 \%)$, principalmente entre as adolescentes e nas dermatites localizadas na face, pescoço e axilas, relacionadas com perfumes, cosméticos e desodorizantes. ${ }^{11}$

Os conservantes foram causa de DCA relacionados com cosméticos e medicamentos. Atualmente e perante uma epidemia europeia de dermatites de contacto à metilisotiazolinona, ${ }^{30-32}$ encontrámos também reações relevantes em crianças devido à utilização de cosméticos e produtos de higiene pessoal. Destacamos um caso suspeito de reação adversa ao etanercept em que os testes permitiram atribuir as lesões eczematosas generalizadas à metilisotiazolinona identificada no gel de higiene pessoal.

O timerosal, conservante outrora usado frequentemente em vacinas, gotas oftálmicas e soluções para lentes de contacto, é um alergénio comum mas raramente relevante, tal como noutros estudos. A progressiva eliminação do mercado aliada à baixa relevância das reacções levou à sua exclusão da série básica em muitos países ${ }^{10,12}$ e também em Portugal.

A DCA a medicamentos tópicos, observada em oito casos, em nenhum delesidentificada com os alergénios da série básica, veio demonstrar a importância de testar os "produtos próprios". Apesar do número de casos de DCA por medicamentos tópicos ter vindo a reduzir progressivamente, sobretudo no adulto, ${ }^{33}$ eles representaram ainda uma percentagem significativa em idade pediátrica (7,3\%).

Nos exantemas medicamentosos, a percentagem de reações positivas $(30,8 \%)$ aproxima-se muito do observado nos adultos, identificando-se fármacos habitualmente referidos como capazes de ocasionar testes positivos: anti-epiléticos (carbamazepina) e antibióticos (flucloxacilina, cefotaxima e vancomicina). ${ }^{34}$

Os casos de reações de hipersensibilidade a medicamentos e outros também não identificados pela série básica de alergénios, sugerem que o conjunto de alérgenios incluídos nesta série não estará tão bem adequado à idade pediátrica.

\section{CONCLUSÃO}

Atualmente, as crianças são expostas a uma grande variedade de alergénios ainda numa idade muito precoce, o que pode tornar a DCA uma patologia cada vez mais frequente na idade pediátrica. A utilização mais alargada e consensual dos testes epicutâneos como um método de diagnóstico útil e imprescindível nestas idades tem vindo a permitir identificar mais casos que anteriormente não seriam diagnosticados. A maioria dos autores concorda que as crianças toleram os testes epicutâneos na mesma concentração dos adultos e que estes testes são fiáveis e seguros em idade pediátrica. ${ }^{1,2}$

O níquel continua a ser um alergénio bastante prevalente, reforçando a ideia de que é necessário implementar regras mais apertadas quanto ao seu uso em artigos que entram em contacto direto com a pele durante longos períodos de tempo.

Conflitos de interesse: Os autores declaram não possuir conflitos de interesse.

Suporte financeiro: $O$ presente trabalho não foi suportado por nenhum subsídio ou bolsa.

Confidencialidade dos dados: Os autores declaram ter seguido os protocolos do seu centro de trabalho acerca da publicação dos dados de doentes.

Protecção de pessoas e animais: Os autores declaram que os procedimentos seguidos estavam de acordo com os regulamentos estabelecidos pelos responsáveis da Comissão de Investigação Clínica e Ética e de acordo com a Declaração de Helsínquia da Associação Médica Mundial

Conflicts of interest: The authors have no conflicts of interest to declare.

Financing Support: This work has not received any contribution, grant or scholarship.

Confidentiality of data: The authors declare that they have followed the protocols of their work center on the publication of data from patients.

Protection of human and animal subjects: The authors declare that the procedures followed were in accordance with the regulations of the relevant clinical research ethics committee and with those of the Code of Ethics of the World Medical Association (Declaration of Helsinki).

\section{REFERÊNCIAS}

1. Torres T, Machado S, Selores M. Dermatite de contacto alérgica em criança e adolescentes - estudo retrospectivo de 11 anos. Trab Soc Port Dermatol Venereol. 2009; 67:253-8.

2. Johansen DJ, Aalto-Korte K, Agner T, Andersen KE, Bircher A, Bruze M, et al. European Society of Contact Dermatitis guideline for diagnostic patch testing - recommendations on best practice. Contact Dermatitis. 2015; 73:195-221.

3. Diepgen $T L$, Ofenloch RF, Bruze M, Bertuccio P, Cazzaniga S, Coenraads PJ, et al. Prevalence of contact allergy in the general population in different European regions. Br J Dermatol. 2016; 174: 319-29.

4. Machovcova A. The frequency of contact allergy in 


\section{Artigo Original}

children and adolescents in the Czeck Republic. Acta Dermatovenereol Croat. 2012; 20: 75-9.

5. Kwan JM, Jacob SE. Contact Dermatitis in the atopic child. Pediatr Ann. 2012; 10:422-3; 426-8.

6. Peiser M, Tralau T, Heidler J, Api AM, Arts JH, Basketter DA, et al. Allergic contact dermatitis: epidemiology, molecular mechanisms, in vitro methods and regulatory aspects. Cell Mol Life Sci. 2012; 69:763-81.

7. Almeida LS, Freire T. Metodologia da Investigação em Psicologia e Educação. $3^{a}$ Edição Revista e Ampliada. Braga: Psiquilíbrios; 2003.

8. Gonçalo S, Gonçalo M, Azenha A, Barros MA, Bastos AS, Brandão FM, et al. Allergic contact dermatitis in children. Contact Dermatitis 1992: 26:112-5.

9. Simonsen $A B$, Deleuran $M$, Johansen JD, Sommerlund $M$. Contact allergy and allergic contact dermatitis in children - a review of current data. Contact Dermatitis. $2011 ; 65: 254-65$.

10. Militello G, Jacob SE, Crawford GH. Allergic contact dermatitis in children. Curr Opin Pediatr. 2006; 18:385-90.

11. Mortz CG, Lauritsen JM, Bindslev-Jensen C, Andersen KE. Contact allergy and allergic contact dermatitis in adolescents: prevalence measures and associations. The Odense Adolescence Cohort Study on Atopic Diseases and Dermatitis. Acta Derm Venereol. 2002; 82:352-8.

12. Pigatto P, Martelli A, Marsili C, Fiocchi A. Contact dermatitis in children. Ital J Pediatr. 2010; 36:2.

13. Nijhawan Rl, Matiz C, Jacob SE. Contact dermatitis: from basics to allergodromes. Pediatr Ann. 2009; 38:99-108.

14. de Waard-van der Spek FB, Andersen KE, Darsow U, Mortz CG, Orton D, Worm M, et al. Allergic contact dermatitis in children: which factors are relevant? (review of the literature). Pediatr Allergy Immunol. 2013: 24:321-9.

15. Milingou M, Tagka A, Armenaka M, Kimpouri K, Kouimintzis $D$, Katsarou A. Patch tests in children: a review of 13 years of experience in comparison with previous data. Pediatr Dermatol. 2010; 27:255-9.

16. Krecisz B, Chomiczewska D, Cezary P, Kiec-Swierczynska M. Contact allergy to metals in adolescents. Nickel release from metal accessories 7 years after the implementation of the EU Nickel Directive in Poland. Contact Dermatitis. 2011; 67:270-6.

17. Schena D, Papagrigoraki A, Tessari G, Peroni A, Sabbadini C, Girolomoni G. Allergic contact dermatitis in children with and without atopic dermatitis. Contact Dermatitis, 2012; 23:275-80.

18. Imbesi S, Minciull PL, Isola S, Gangemi S. Allergic contact dermatitis: Immune system involvement and distinctive clinical cases. Allergol Immunopathol. 201 1; 39:374-7.

19. Barros MA, Baptista A, Correia M, Azevedo F. Patch testing in children: a study of 562 children. Contact Dermatitis. 1991; 25:156-9.

20. Jacob SE, Brod B, Crawford GH. Clinically relevant patch test reactions in children - a United States based study. Pediatr Dermatol. 2008; 25:520-7.

21. Hogeling $M$, Pratt $M$. Allergic contact dermatitis in children: the Ottawa hospital patch-testing clinic experience, 1996 to 2006. Contact Dermatitis. 2008;19:86-9.

22. Teixeira V, Coutinho I, Gonçalo M. Alergia de contacto a metais num período de 20 anos no centro de Portugal: Implicações das Directivas Europeias. Acta Med Port. 2014; 27:295-303.

23. Garg S, Thyssen JP, Uter W, Johanssen JD, Menné T, BeIloni Fortina $A$, et al. Nickel allergy following European Union regulation in Denmark, Germany, Italy and the U.K. Br J Dermatol. 2013; 169:854-8.

24. Kickinger-Lörsch A, Bruckner T, Mahler V. Nickel and cobalt release from metal alloys of tools-a current analysis in Germany. Contact Dermatitis. 2015 73:289-95.

25. Van Baelen A, Kerre S, Goossens A. Allergic contact cheilitis and hand dermatitis caused by toothpaste. Contact Dermatitis. 2016; 74:187-9.

26. Borrego LM, Melo H. Dermatite de contacto na criança. Rev Port Imunoalergol. 2014; 22:153-4.

27. Morton LM, Masterpol KS. Contact Dermatitis in Children. Contact Dermatitis. Philadelphia: In Tech; 2011.

28. Rachmawati D, Bontkes HJ, Verstege MI, Muris J, von Blomberg BM, Scheper RJ, et al. Transition metal sensing by Toll-like receptor-4: next to nickel, cobalt and palladium are potent human dendritic cell stimulators. Contact Dermatitis. 2013; 68:331-8.

29. Haluk Akar H, Adatepe S, Tahan F, Solmaz I. Hair dyes and temporary tattoos are a real hazard for adolescents? Eur Ann Allergy Clin Immunol. 2014; 46:35-7.

30. Gonçalo M, Goossens A. Whilst Rome burns: the epidemic of contact allergy to methylisothiazolinone. Contact Dermatitis. 2013; 68:257-8.

31. Gameiro A, Coutinho I, Ramos L, Gonçalo M. Methylisothiazolinone: second 'epidemic' of isothiazolinone sensitization. Contact Dermatitis. 2014; 70:242-3.

32. Schwensen JF, Uter W, Bruze M, Svedman C, Goossens A, Wilkinson $M$, et al. The epidemic of methylisothiazolinone: a European prospective study. Contact Dermatitis. 2017; 76:272-9.

33. Gilissen L, Goossens A. Frequency and trends of contact allergy to and iatrogenic contact dermatitis caused by topical drugs over a 25 -year period. Contact Dermatitis. 2016; 75:290-302.

34. Gonçalo M, Bruynzeel D. Patch testing in drug eruptions. Contact Dermatitis. Berlin: Springer-Verlag; 2011. 